\title{
GASA Tuned Optimal Fuzzy Regulator for AGC of an Interconnected Power System
}

\author{
Ibraheem, Naimul Hasan and Omveer Singh \\ Faculty of Engg. \& Tech., Jamia Millia Islamia \\ New Delhi -110025 India
}

\begin{abstract}
An optimal fuzzy AGC regulator design based on GASA tuning technique for interconnected power system is proposed in this paper. The investigations with the GASA tuned optimal fuzzy regulator are carried out on a two area interconnected power system consisting of power plants with different characteristics. Power system area- 1 is having plants with reheat thermal turbines whereas area-2 has plants with hydro turbines. The dynamic response plots are obtained for $1 \%$ load disturbance in hydro area. The dynamic response plots are obtained for various system states with the implementation of conventional integral, fuzzy logic and GASA based AGC regulators designed in the study. The GASA technique is applied to tune the gains of fuzzy logic based AGC regulators also. The simulation results demonstrate superiority of GASA technique based optimal fuzzy AGC regulators over the others.
\end{abstract}

\section{Keywords}

Automatic generation control (AGC), Fuzzy logic regulator (FLR), Area control error (ACE), Genetic Algorithm-Simulated Annealing (GASA), interconnected power system

\section{INTRODUCTION}

The overall aim of a power system engineer is to provide a good quality of electricity to the consumers. For this, an electric power system must be maintained at the desired operating level characterized by nominal frequency, voltage profile and load flow configuration. It is kept in this nominal state by close control of real and reactive powers generated in the controllable source of the system.

For the maintenance of nominal state of the system, the generation changes must be made to match the load perturbation. The control of a large interconnected power system, in order to achieve an exact matching of the generation to load at nominal state, is a challenging problem. It is so since in a power system the load continually changes and the system generation, responding to control signals, chases the load with the transient unbalance of the load and generation reflected in frequency and voltage variations. Effective control strategies need to be evolved to keep the system performance within sufficiently small tolerance levels. For small perturbations, a mismatch in real power balance affects primarily the system frequency but leaves the bus voltage magnikudis essentially unaffected and similarly a mismatch in reactive power imbalance affects only the bus voltage magnitude, but leaves the system frequency unaffected. In view of this, the real power-frequency (P-f) control problem and reactive power-voltage $(\mathrm{Q}-\mathrm{V})$ control problems are treated as two independent or decoupled control problems for all practical purposes. Frequency is an index of the health of a power system and hence it is desirable to achieve better frequency constancy than is obtained by the speed governing system alone. In an interconnected power system it is desirable to maintain the tie line power flow at scheduled level irrespective of the load changes in any area. To accomplish this, the position of main steam valves or hydro gates is controlled in accordance with a suitable control strategy, which in turn controls the real power output of electrical generators. The control of real power output of electric generators in this way is termed as Automatic generation Control (AGC).

A large interconnected power system is divided into a large number of control areas for automatic generation control. A control area is defined as a power system, a part of the system, or a combination of the systems to which a common generation control scheme is applied. Each control area comprises an equivalent generator, equivalent turbine, and equivalent excitation system and equivalent governor.

The electrical connections within each control area are very strong as compared to ties with the neighboring areas. All the generators in a control area swing in unison or coherently and it is characterized by a single frequency. It is necessary to consider as many control areas as the number of such coherent groups. In normal steady-state operation each control area of a power system should strive to meet its own load demand and in addition participate in regulating the frequency of the system. AGC is defined as the regulation of the power output of electric generators within a prescribed area in response to a change in system frequency and/ or tie-line loading, or the relation of these to each other, so as to maintain the scheduled system frequency and/or the established interchange with other areas within predetermined limits [1-3].

The conventional AGC regulators have been found inadequate to deal with complex integrated power system control problems because, they works on the trial and error principle [4]. Therefore, intelligent techniques have taken over the lead to overcome the weaknesses associated with these conventional techniques. These intelligent technique based AGC regulators are adaptive and robust in nature. Due to these novel features, intelligent technique based regulators have been identified as one of the most justifiable option to deal complex system like interconnected power systems. The area of intelligent techniques include the concepts like artificial neural network (ANN), fuzzy logic (FL), genetic algorithm (GA), and simulated annealing (SA) etc. A number of research articles have been appeared in the literature from time to time dealing with the design of AGC regulators for isolated/ interconnected power systems. In the ensuing paragraph few of them are reviewed.

An algorithm based on fuzzy logic concept to determine the optimal parameters for the extended integral control scheme is proposed by Moon and Ryu in [4]. The fuzzy rule base was developed considering change of frequency and its rate as inputs. A set of decision rules established to relate input signals to the decaying factor. Whereas; Chang and Fu [5] used FL algorithm for tuning the gains of area load frequency controllers for a multi area power system. Rerkpeedapong and Feliachi [6] designed fuzzy rules to design load frequency regulators of generating units providing regulation and load following services in the multi-area power system model. 
Besides the FL concept, the power engineers have paid a due attention to consider GA as an intelligent tool to design optimal AGC regulators due to its inherent features to get global minima for an optimization problem. Aditya [7] had demonstrated the benefits of GA applications in handling the AGC problem over the conventional schemes. In [8], Golpira addressed the necessity of incorporation of system non-linearities like; generation rate constraint (GRC), dead band, and time delay imposed to the power system by governor-turbine, filters, thermodynamic process, and communication channels while designing the AGC scheme for the power systems. In the work reported by him, GA is used to compute the decentralized control parameters to achieve an optimum operating point. A 3-control area power system is considered as a test system, and the closed-loop performance is examined in the presence of various constraints scenarios. Also AGC schemes based on evolutionary techniques are proposed by Patel [9]. The results of the study were compared with that obtained with conventional techniques.

Simulated annealing (SA) technique is applied by Bensenouci [10] to design optimal AGC schemes for power systems. Three optimum adaptive variable-structures are proposed and their applications to AGC of an interconnected power system, namely, optimum dither (DT), optimum PI, and optimum variable input (VI) control have been demonstrated. The main objective of the work is to maintain robustness while reducing the chattering effect in variable structure control (VSC) at the same time fulfilling the AGC requirements. Contrary to the widely used trial-and-error technique, these approaches allow the determination of the controller gains systematically and adaptively in a step-wise fashion using simulating annealing, a well known robust optimization technique. The VSC gains computation problem was converted to an optimization problem using a suitable cost function that was based on Lyapunov theory of VSC.

Moreover, these regulators are found suitable for optimizing the feedback gains of an AGC regulator in the power system but they could not track the best solution for the AGC problem and therefore are associated with certain limitations[11]. To utilize the salient features of various types of these techniques, idea of designing AGC regulators based on a compatible combination of these techniques to suit the area of application was mooted in the frame of mind of power engineers.

Following this, Das and Patvardhan [12] have applied evolutionary algorithms (EA) in the field of power system. The hybridization of EA techniques has been demonstrated as a good option for the system parameters optimization. In [13], Li and Du have reported a work on multi area GA based fuzzy logic control scheme for AGC of power system. The fuzzy decision table is used with GA based looking up algorithm for AGC system performance optimization. The AGC scheme was capable to treat nonlinear element in AGC, such as generator rate constraint (GRC) and saturation easily. Juang and $\mathrm{Lu}$ [14] demonstrated that a fuzzy system can be used to adaptively decide integral and proportional integral AGC regulator gain according to the ACE and their changes. To improve the design performance of fuzzy system, a new GA approach using elitist strategy combined with similarity measure on relatives between individuals is used.

The gains of optimal integral, proportional integral and PID AGC regulators are computed by hybrid genetic algorithm-simulated annealing (GASA) technique and Hybrid Particle Swarm Optimization (HBPSO) technique for nominal values of area input parameters and optimal transient responses of area frequency deviations in terms of settling times, undershoots, overshoots and area frequency deviations as output with incremental increase of area load for interconnected three equal generating areas $[15,16]$. Optimal PID gains as determined by hybrid GASA technique are found to be more globally optimal than those determined by GA method. For off-nominal input parameters, the inspection of transient responses as determined by fast acting Sugeno fuzzy logic technique reflect the superiority of GASA based optimized gains, specially for PID control.

In most these works reported so far, the power system models considered are of same characteristics and identical. However, in practical situations, this ideal situation is not available. The power systems areas may be of different characteristics. This paper is dedicated to consider a two area interconnected power system interconnected via EHVAC transmission lines consisting of plants with reheat thermal and hydro turbines. The optimal AGC regulators are designed based on fuzzy logic concept and the gains AGC regulators are optimized using GASA technique. The various AGC regulators are designed and implemented on power system model under 0.01 p.u.MW load perturbation in the hydro area. The power system dynamic performance has been studied by investigating the response plots of the disturbed areas.

\section{MULTI-AREA POWER SYSTEM MODEL UNDER INVESTIGATION}

A two area interconnected power system consisting of different plants with reheat thermal and hydro turbines are considered for the study. The transfer function model of interconnected hydro-thermal power system is shown in Fig.4.

The integral square error criterion is adopted for developing objective function for optimization of parameters. This can be defined as follows [9]

$$
\mathrm{ISE}=\int_{0}^{\infty} \mathrm{e}^{2}(\mathrm{t}) \mathrm{dt}
$$

where; ISE refers to Integral Square Error, e corresponds to error. In ISE, only error is considered and therefore no weight is given to time span of error.

For the present study; the formulation of ISE can be given as:

$$
\mathrm{ISE}=\int_{0}^{30} \mathrm{ACE}^{2} \mathrm{dt}
$$

where ACE represents area control error of a power system area and is defined as:

$\mathrm{ACE}_{1}=\mathrm{B}_{1} \Delta \mathrm{F}_{1}+\Delta \mathrm{P}_{\text {tie, }}$ for area 1

and for area $2, \mathrm{ACE}_{2}=\mathrm{B}_{2} \Delta \mathrm{F}_{2}+\Delta \mathrm{P}_{\text {tie, }}$

The ACE of respective areas is taken as the input to the integral controllers which can be expressed as:

$$
\mathrm{U}_{\mathrm{i}}=-\int \mathrm{K}_{\mathrm{i}}(\mathrm{ACE}) \mathrm{dt}
$$

The simulation study has been performed using standard MATLAB software. The SIMULINK TOOLBOX is also used to achieve the dynamic response plots for various system states.

\section{FUZZY LOGIC REGULATOR}

The structure of fuzzy logic based AGC regulator is described in Fig. 1. It consists of a fuzzy logic AGC regulator cascaded with conventional integrator. The integral regulator has one input signal, namely, area control error (ACE) of the system which exhibits the deviation in frequencies of interconnected power system areas and change in tie-line exchanges, and then the output signal (Y) of integrator block becomes the input signal to fuzzy logic based regulator. Finally, the output signal from the fuzzy logic based regulator called the control signal $\left(\mathrm{U}_{\mathrm{i}}=\int \mathrm{ACE} \mathrm{dt}\right)$ is used for performing the duties of AGC. Control signal gain scheduling of a nonlinear power system is a very important and effective process of controlling [5]. It is generally done when the system dynamics and operating conditions are available, and for 
which a single linear time-invariant model is not adequate to handle.

For the present study, seven triangular membership functions of the fuzzy logic based AGC regulator (FLR) are used. In the FLR, one input and one output is selected in the fuzzy inference engine. The FLR has seven rules for each ACE.

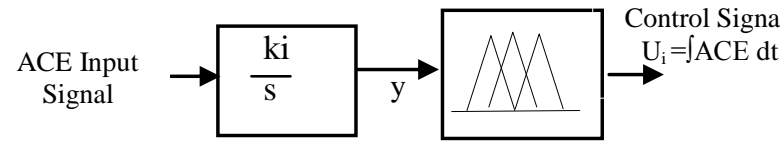

Integral Regulator Fuzzy Logic Regulator

Fig.1 Structure of the Fuzzy Logic based AGC Regulator

(i)

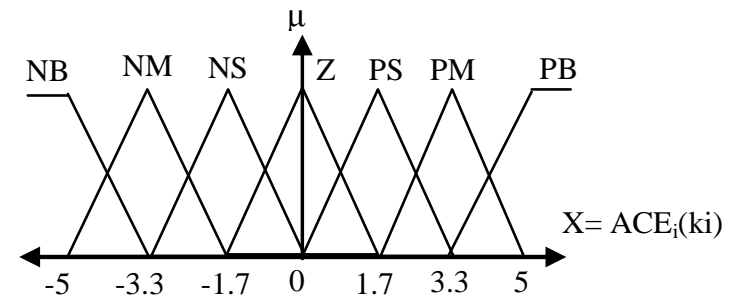

(ii)

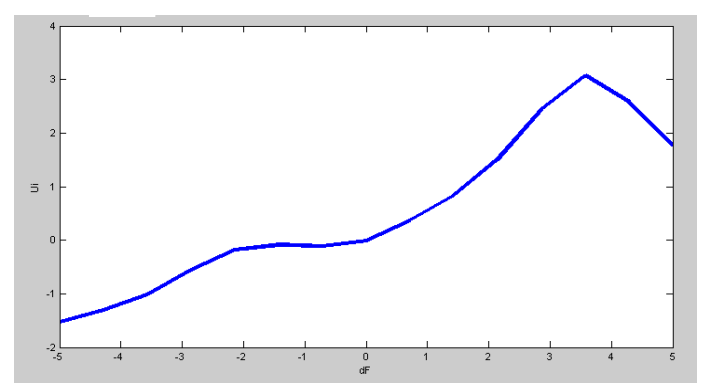

Fig.2 Membership functions for FLR of (i) $\mathrm{ACE}_{\mathrm{i}}(\mathrm{ki})$ (ii) Surface view of input $\&$ output

In the fuzzy technique; triangular membership functions shapes of the derivative error and gains according to $\mathrm{Y}$ are chosen to be identical for the FLR. However, its horizontal axis limits are taken different values for calculating the regulator output. There are two regulators, $\mathrm{FLR}_{\mathrm{t} 1}$ and $\mathrm{FLR}_{\mathrm{h} 2}$. The membership functions of fuzzy logic for ACE are shown in Fig. 2(i). Each of the regulators has the Negative Big NB, Negative Medium NM, Negative Small NS, Zero Z, Positive Small PS, Positive Medium PM, Positive Big PB fuzzy rules with respect to ACE are shown in [Table1] and the view of rules for fuzzy logic regulator under investigation is presenting in Fig. 2(ii). $\mu$ is the weights for the control signals. The rules are interpreted as follows:

If $\Delta \mathrm{F}$ is $\mathrm{NM}$ then the output of the regulator is PM.

Output control $U_{i}$ characteristic of the fuzzy regulator represent a conditional relationship between input signal ACE and output signal $U_{\mathrm{i}}$ in Fig.2 (ii). In this graph; $x$ level shows the regulator input and y level present output of the fuzzy regulator. Rule base are defined in the range of $[-5,5]$.

Table 1: Fuzzy Rule-base system

\begin{tabular}{|l|c|}
\hline \multicolumn{2}{|c|}{ Fuzzy Rules for FLR } \\
\hline Rule1 & If(ACE is NB) then $\left(\mathrm{U}_{\mathrm{i}}\right.$ is $\left.\mathrm{PB}\right)$ \\
\hline Rule2 & If(ACE is NM) then $\left(\mathrm{U}_{\mathrm{i}}\right.$ is $\left.\mathrm{PM}\right)$ \\
\hline Rule3 & If(ACE is NS) then $\left(\mathrm{U}_{\mathrm{i}}\right.$ is $\left.\mathrm{PS}\right)$ \\
\hline Rule4 & If(ACE is $\mathrm{Z})$ then $\left(\mathrm{U}_{\mathrm{i}}\right.$ is $\left.\mathrm{Z}\right)$ \\
\hline Rule5 & If(ACE is PS) then $\left(\mathrm{U}_{\mathrm{i}}\right.$ is NS) \\
\hline Rule6 & If(ACE is $\mathrm{PM})$ then $\left(\mathrm{U}_{\mathrm{i}}\right.$ is $\left.\mathrm{NM}\right)$ \\
\hline Rule7 & If(ACE is $\mathrm{PB})$ then $\left(\mathrm{U}_{\mathrm{i}}\right.$ is $\left.\mathrm{NB}\right)$ \\
\hline
\end{tabular}

\section{GASA TUNED OPTIMAL FUZZY LOGIC REGULATOR DESIGN}

The Genetic Algorithm - Simulated Annealing (GASA) technique can be viewed as an adaptation with suitable modifications of the Evolutionary Simulated Annealing Heuristic [12] for function optimization. It has been designed with a real coded string representation of the chromosomes on which the genetic blend crossover operator is used.

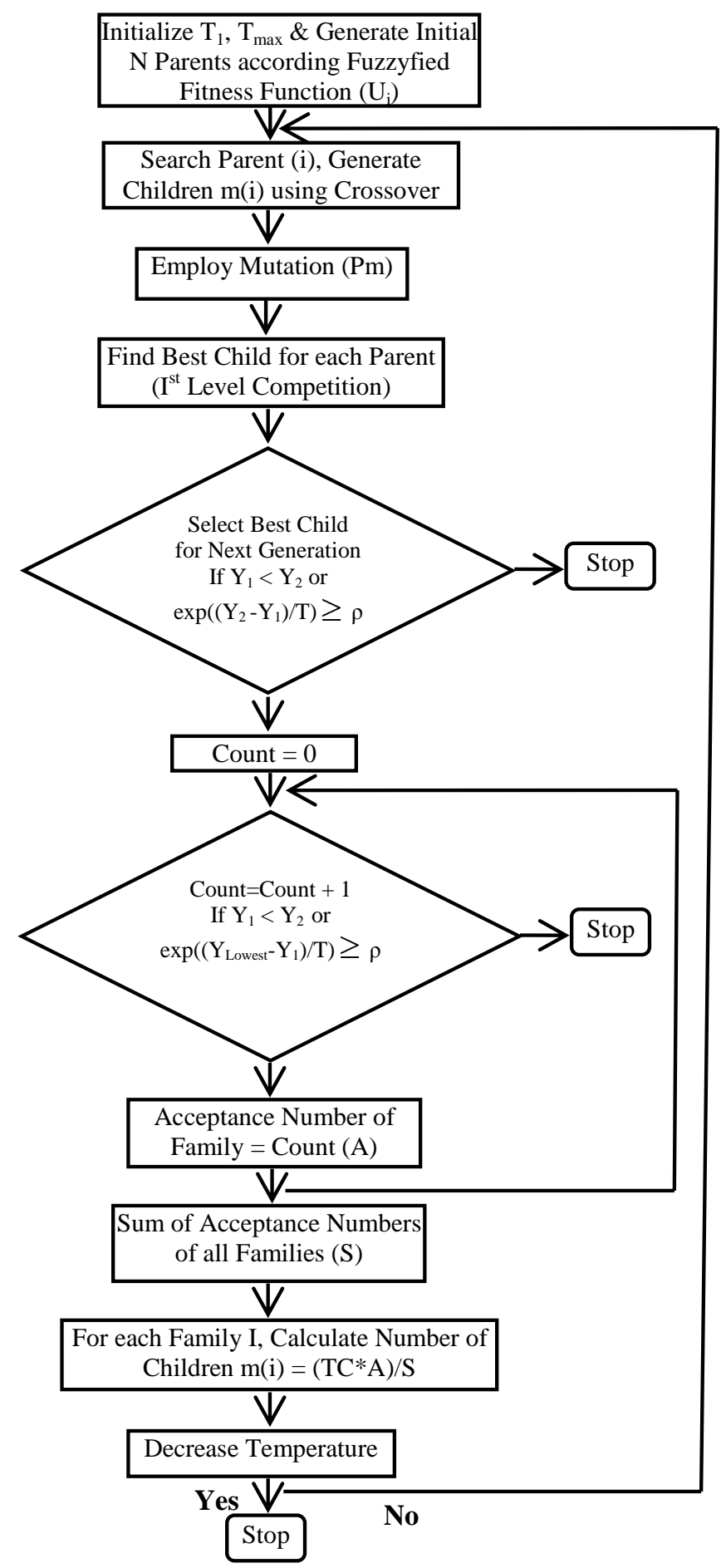

Fig.3 Flowchart for a GASA tuned Fuzzy Logic Regulator

This proposed approach incorporates SA in the selection process of GA. This strategy helps in maintaining diversity in the search process and avoiding getting stuck to local optimum as sometimes has been witnessed in case of applications of GA. The proposed 
algorithm of GASA for the adaption of fuzzy fitness function can be represented in the form of a flow chart.

The solution string comprises of fitness function of ISE parameters from the fuzzy logic regulators. The numerical values of scaling factors $\mathrm{X}$ and $\mathrm{Y}$ are set 5 for un-tuned regulators whereas its values are found by GASA approach for tuned fuzzy regulators. GASA heuristic employs blend crossover and a mutation operator suitable for real number representation to provide a better search capability.

In the application of this concept in the tuning of parameters for optimal fuzzy AGC scheme, the objective is to optimize the fitness function values of the FLR augmented with penalty terms. The heuristic is quite general and various aspects like nonlinear, discontinuous functions and constraints are easily incorporated as per requirements [15].

The solution string comprises of the fitness function of area control error of the interconnected power system. A real string representation is chosen. Its size is chosen as 100 . Following are the implementation details of the algorithm:

4.1 Crossover: For each parent $i$, mates are selected from the other parents at random and crossover is applied to generate $\mathrm{m}(\mathrm{i})$ children. To start with, m(i) is fixed same for all the parents. This number is changed as the search progresses as explained in the subsequent steps.

The blend crossover operator (BLX- $\alpha$ ) based on the theory of interval schemata is employed in this study. BLX- $\alpha$ operates by randomly selecting a point in the range (p1- $\alpha(\mathrm{p} 2-\mathrm{p} 1)$, $\mathrm{p} 2+\alpha(\mathrm{p} 2-\mathrm{p} 1))$, where $\mathrm{p} 1$ and $\mathrm{p} 2$ are the two parent points and $\mathrm{p} 1<\mathrm{p} 2$.

4.2 Fitness Evaluation: The objective here is to minimize the ACE of the two areas. The fitness function is taken as the summation of the absolute values of the three at every discrete time instant in the simulation. An optional penalty term is added to take care of the transient response specifications viz. upper bound on the regulator output, system frequency and tie-line flows, settling time etc.

4.3 Selection: The best child (the child with minimum objective value) out of the children generated from the same parent is found. The best child then competes with it's parent to survive in the next generation. If the best child is better than it's parent, it is accepted as a parent in the next generation. If the best child is worse than it's parent then there is the Boltzmann criterion that the child be accepted as given below. The selection process is inspired from the SA mechanism and allows a bad move to be selected in the optimization process with a probability.

4.4 Boltzmann Criterion: As in SA, the selection of temperatures is such that initially the probability of acceptance of a bad move, i.e., accepting the best child as the parent for next generation when it is worse than the current parent, is high (approximately 1) but as the temperatures are successively lowered this probability is decreased till, at the end, the probability of accepting a bad move is negligible (approximately 0). Such a strategy enables the technique to seek the global optimum without getting stuck in any local optimum [12]. The initial and final temperatures are calculated as follows:

A bad move is accepted according to the Boltzmann Criterion. Initially the probability of accepting a bad move is approximately one, i.e.

$$
\exp \left(-\Delta X_{\text {average }} / \mathrm{T}^{1}\right)=0.99
$$

and finally

$$
\exp \left(-\Delta X_{\text {average }} / \mathrm{T}^{\mathrm{MAXIT}}\right)=0.0001
$$

Therefore; $T^{1}=-\Delta X$ average $/ \log (0.99)$

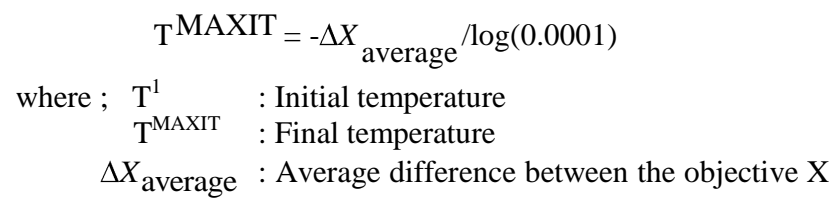

for any two neighborhood points in the search space. This average is calculated over a number of chromosomes.

4.5 Cooling Schedule: Temperature is cooled down with the iterations as under [12];

$$
\begin{aligned}
& \mathrm{T}^{\mathrm{k}+1}=\mathrm{T}^{\mathrm{k}} /\left(1+\beta \cdot \mathrm{T}^{\mathrm{k}}\right) \\
& \text { Where; } \beta=\left(\mathrm{T}^{1}-\mathrm{T}^{\mathrm{MAXIT}}\right) /\left(\mathrm{T}^{1} \cdot \mathrm{T}^{\mathrm{MAXIT}}\right. \text {.(MAXIT-1)) } \\
& \text { and superscript } \mathrm{k} \text { represents the iteration count. }
\end{aligned}
$$

4.6 Acceptance Number: The number of children generated in the next generation is proportional to a parameter called the acceptance number. This number provides a measure of the goodness of the solutions in the vicinity of the current parent. The number is computed by sampling the search space around the current parent and counting the number of good samples out of the total samples as per steps of flow chart. This strategy enables the algorithm to focus search on the better regions of the search space. The GASA used here employs direct manipulation of the fuzzy parameters.

\section{SIMULATION RESULTS}

The system dynamic response plot are obtained for various system states like $\Delta \mathrm{Ft}_{1}, \Delta \mathrm{Fh}_{2}, \Delta \mathrm{Pg}_{\mathrm{t} 1}, \Delta \mathrm{Pg}_{\mathrm{h} 2}, \Delta \mathrm{P}_{\text {tie }}$ and ACE to investigate the effectiveness of conventional integral (IR), fuzzy logic based (FLR) and genetic algorithm-simulated annealing tuned (GASA) optimal AGC regulators. The optimal AGC regulators are applied to two-area interconnected power system model considering $1 \%$ load disturbance in hydro area. These response plots are shown in Figs.5-10.

The investigations of these response plots reveal that implementation of GASA based AGC regulators reduce the magnitude of first peak of the response to a great extent and number of oscillations considerably as compared to that obtained with IR and FLR. The settling time of all the responses has also reduced appreciably in case of GASA based optimal AGC regulators. These investigations are depicted in Table 2. However, a zero steady state error is ensured with the implementation of all the three types of AGC regulators.

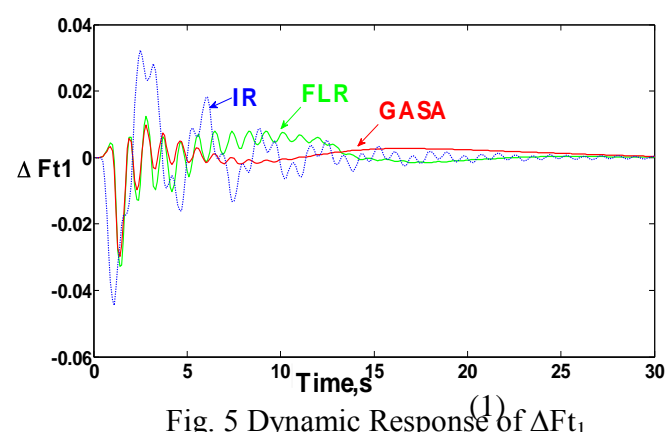




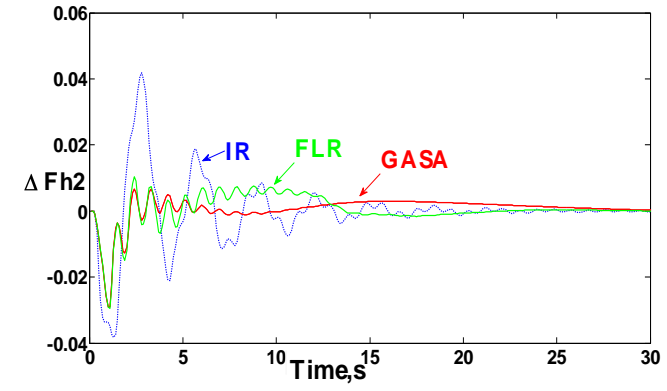

Fig. 6 Dynamic Response of $\Delta \mathrm{Fh}_{2}$

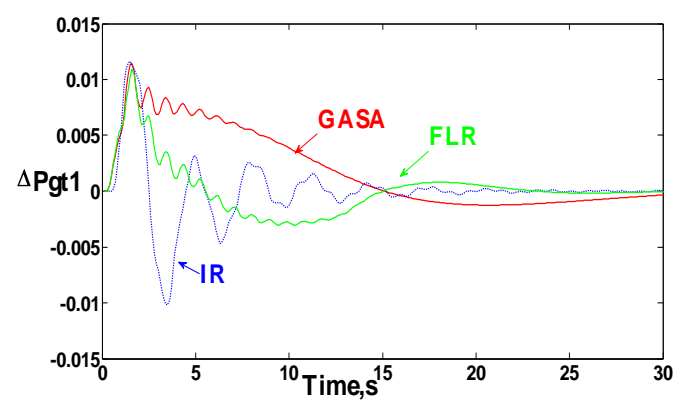

Fig. 7 Dynamic Response of $\Delta \mathrm{Pg}_{\mathrm{t} 1}$

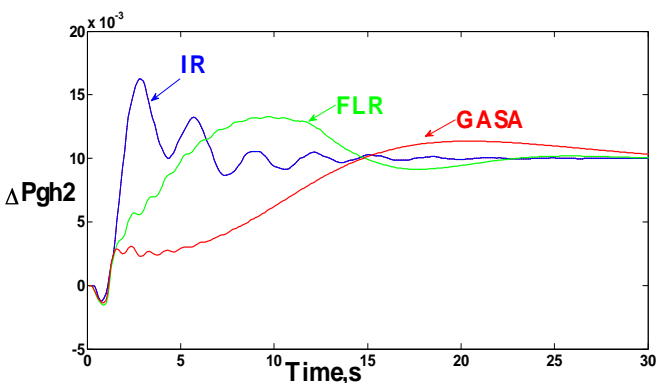

Fig. 8 Dynamic Response of $\Delta \mathrm{Pg}_{\mathrm{h} 2}$

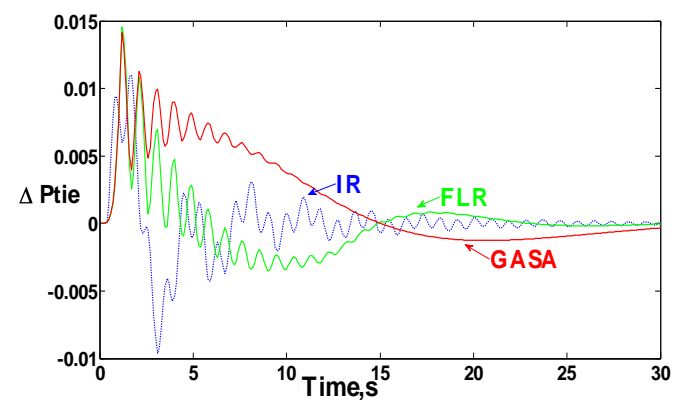

Fig. 9 Dynamic Response of $\Delta \mathrm{P}_{\text {tie }}$

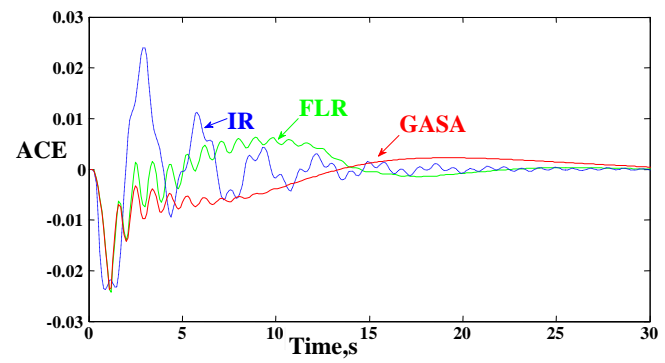

Fig.10 Dynamic Response of ACE
Table 2:

\begin{tabular}{|c|c|c|}
\hline $\begin{array}{c}\text { Type of } \\
\text { Regulator }\end{array}$ & $\begin{array}{c}\text { Peak Overshoot } \\
\left(\Delta \mathbf{F}_{\mathbf{2}}\right), \mathbf{p . u .}\end{array}$ & $\begin{array}{l}\text { Settling Time } \\
\left(\Delta \mathbf{F}_{\mathbf{2}}\right), \mathbf{s e c}\end{array}$ \\
\hline IR & 0.03 & 30 \\
\hline FLR & 0.13 & 14.1 \\
\hline GASA & 0.01 & 12.5 \\
\hline
\end{tabular}

\section{CONCLUSION}

The GASA tuned optimal fuzzy AGC regulators are proposed for a two-area interconnected hydro-thermal power system interconnected via EHVAC transmission line. The investigations carried out in the study show that the proposed GASA tuned optimal fuzzy AGC regulators offers an ameliorated system dynamic performance when implemented under system load perturbation conditions. The proposed AGC regulators are very effective in suppressing oscillations associated with system dynamic responses while simultaneously improving the settling time.

\section{APPENDIX (A)}

Description of Abbreviations

$\Delta \mathrm{F}_{\mathrm{i}}$ : Incremental Change in Frequency

subscript referring to area $\left(\mathrm{i}=\mathrm{t}_{1}, \mathrm{~h}_{2}\right)$

$\Delta \mathrm{P}_{\mathrm{gi}}$ : Incremental Change in Generator Power Output

$\Delta \mathrm{P}_{\mathrm{di}}$ : Incremental Change in Load Demand (p.u.MW/Hz)

$\Delta \mathrm{P}_{\mathrm{ci}}$ : Incremental Change in Load Demand (p.u.MW/Hz)

$\Delta \mathrm{X}_{\mathrm{gi}}$ : Incremental Change in Governor Valve Position

$\Delta \mathrm{Xgh}_{\mathrm{h} 2}$ : Intermediate Output of Hydro Governor

$\Delta \mathrm{P}_{\text {tie }}$ : Incremental Change in Tie-line Power (MW)

$\mathrm{T}_{\mathrm{wi}}$ : Hydro Turbine Time Constant (sec.)

$\mathrm{T}_{1}$ : Hydro Governor Time Constant of Area, $\mathrm{s}$

$\mathrm{T}_{2}, \mathrm{~T}_{3}$ : Time Constants Associated with Hydro Governor

$\mathrm{T}_{\mathrm{p}}$ : Electric System Time Constants

$\mathrm{R}_{\mathrm{i}}$ : Speed Regulation Parameter, Hz/p.u.MW

$\mathrm{K}_{\mathrm{i}}$ : Integral Gain Constant

$\mathrm{T}_{\mathrm{g} 1}$ : Speed Governor Time Constant of Area, $\mathrm{s}$

$\mathrm{K}_{\mathrm{ri}}, \mathrm{T}_{\mathrm{ri}}$ : Reheat Coefficient's \& Reheat Time's

$B_{i} \quad$ : Frequency Bias Constant (p.u.MW/Hz)

ACE : Area Control Error's

$\mathrm{T}_{\mathrm{t} 1}$ : Turbine Time Constants, sec

$\mathrm{K}_{\mathrm{gi}}$ : Speed Governor Gain

$\mathrm{K}_{\mathrm{t} 1}$ : Reheat Thermal Turbine Gain Constant

$\mathrm{T}_{12}$ : Synchronizing Coefficient of AC Tie-line

A, B, C, Г: System Matrices Associated with State, Control, Output and Disturbance Vectors respectively

$\underline{X}, \underline{U}, \underline{Y}, \underline{P d}$ : State, Control, Output and Disturbance Vectors respectively

$\mathrm{T}_{1}, \mathrm{~T}_{\max }$ : Initial and Final Temperatures

$\mathrm{Y}_{1}$ : Objective Value of the Best Child

$\mathrm{Y}_{2}$ : Objective Value of its Parent

$\rho$ : Random Number Uniformly Distributed b/w 0 and 1

TC : Total Number of Children Generated by all the Families

\section{APPENDIX (B)}

Power System Parameters: Nominal system frequency $=50 \mathrm{~Hz}$ $\mathrm{Pr}_{\mathrm{t} 1}=\mathrm{Pr}_{\mathrm{h} 2}=2000 \mathrm{MW}, \mathrm{B}_{\mathrm{t} 1}=\mathrm{B}_{\mathrm{h} 2}=0.425, \mathrm{R}_{\mathrm{t} 1}=\mathrm{R}_{\mathrm{h} 2}=2.4 \mathrm{~Hz} /$ p.u. $\mathrm{MW}, \mathrm{K}_{\mathrm{g} 1}=\mathrm{K}_{\mathrm{g} 2}=1, \mathrm{~T}_{\mathrm{g} 1}=0.08 \mathrm{sec}, \mathrm{K}_{\mathrm{r} 1}=0.5 \mathrm{sec}, \mathrm{T}_{\mathrm{r} 1}=10 \mathrm{sec}, \mathrm{K}_{\mathrm{t} 1}=$ $1, \mathrm{~T}_{\mathrm{t} 1}=0.3 \mathrm{sec}, \mathrm{K}_{\mathrm{pt} 1}=\mathrm{K}_{\mathrm{ph} 2}=120 \mathrm{~Hz} /$ p.u. $\mathrm{MW}, \mathrm{T}_{\mathrm{pt} 1}=\mathrm{T}_{\mathrm{ph} 2}=20$ sec, $\mathrm{a}_{12}=-1,2 \pi \mathrm{T}_{12}=0.545$ p.u.MW, $\mathrm{T}_{1}=5 \mathrm{~s}, \mathrm{~T}_{2}=48.7 \mathrm{~s}, \mathrm{~T}_{3}=5 \mathrm{~s}$, $\mathrm{T}_{\mathrm{w} 2}=1.0 \mathrm{~s}, \Delta \mathrm{Pd}_{\mathrm{h} 2}=0.01$ p.u.MW

GASA parameters:

Population size: 100, Maximum no. of generations (Max Gen): 50, probability: $\operatorname{exp(generation/Max~Gen.),~Mutation~probability:~0.6,~}$ $\alpha=0$. 


\section{REFERENCES}

[1]. Elgerd, O. I. 1982. Electric energy system theory: an introduction.Tata Mc-Graw Hill, 1982.

[2]. Nanda, J. and Kaul, B. L. 1978. Automatic generation control of an interconnected power system. IEE Proc. Generation, Transmission and Distribution, vol. 125, no 5, pp. p 384-390, May 1978.

[3]. Ibraheem and Kumar, P. 2003. Dynamic performance enhancement of hydro power systems with asynchronous tie-lines. Electric Power Components and Systems, vol. 31, no. 7, pp. 605-626, 2003.

[4]. Moon, Young-Hyum and Ryu, Heon-Su. 2001. Fuzzy logic based extended integral control for load frequency control. IEEE Power Engineering Society Winter Meeting, vol. 3, pp. 1289-1293, Feb. 2001

[5]. Chang, C.S. and Fu, W. 1997. Area load frequency control using fuzzy gain scheduling of PI controllers. Electric Power System Research, vol. 42, pp. 145-152, 1997.

[6]. Rerkeedapong, D. and Felachi, A. 2002. Fuzzy rule based load frequency control. IEEE Journal of Power Engineering Society Winter Meeting, vol. 3, no. , pp. 1154-1159, July 2002.

[7]. Aditya, 2003. Design of load frequency controllers using genetic algorithm for two area interconnected hydro power system. Electric Power Components and Systems, 31:1, $81-94,2003$

[8]. Golpira, H., et al. 2011. Application of GA optimization for automatic generation control design in an interconnected power system. Energy Conversion and Management 52 (2011) 2247-2255.
[9]. Patel, R. N., 2007. Application of artificial intelligence for tuning the parameters of an AGC. International Journal of Mathematical, Physical and Engineering Sciences 1;1 2007.

[10].Bensenouci, Ahmed and Ghany. 2007. Step-wise optimum adaptive variable-structure automatic generation control design using simulated annealing”, IEEE Conference, 2007.

[11].Ibraheem, et al. 2005. Recent philosophies of automatic generation control strategies in power systems. IEEE Transaction Power System, vol. 11, no. 3, pp. 346-357, Feb. 2005.

[12].Das, D. B. and Patvardhan, C. 2003. Useful multi-objective hybrid evolutionary approach to optimal power flow. IEE Proceedings-Generation, Transmission and Distribution, Vol. 150, No.3, 275-282, May 2003.

[13].Li, Pingkang and Du, Xiuxix. 2009. Multi-area AGC system performance improvement using GA based fuzzy logic control. International Conference on Electrical Engineering, 2009 .

[14]. Juang, Chia-Feng and Lu, Chun-Feng. 2005. Power System Load Frequency Control by Genetic Fuzzy Gain Scheduling Regulator. Journal of the Chinese Institute of Engineers, Vol 28, No. 6, pp. 1013-1018, 2005.

[15]. Ghoshal, S. P., 2004. Application of GA/GA-SA based fuzzy automatic generation control of a multi-area thermal generating system. Electric Power Systems Research Journal, 70, 115-127, 2004.

[16]. Ghoshal, S. P. and Roy, N. K. 2004. A novel approach for optimization of proportional integral derivative gains in automatic generation control. Australasian Universities Power Engineering Conference (AUPEC 2004). 26-29 September 2004, Brisbane, Australia.

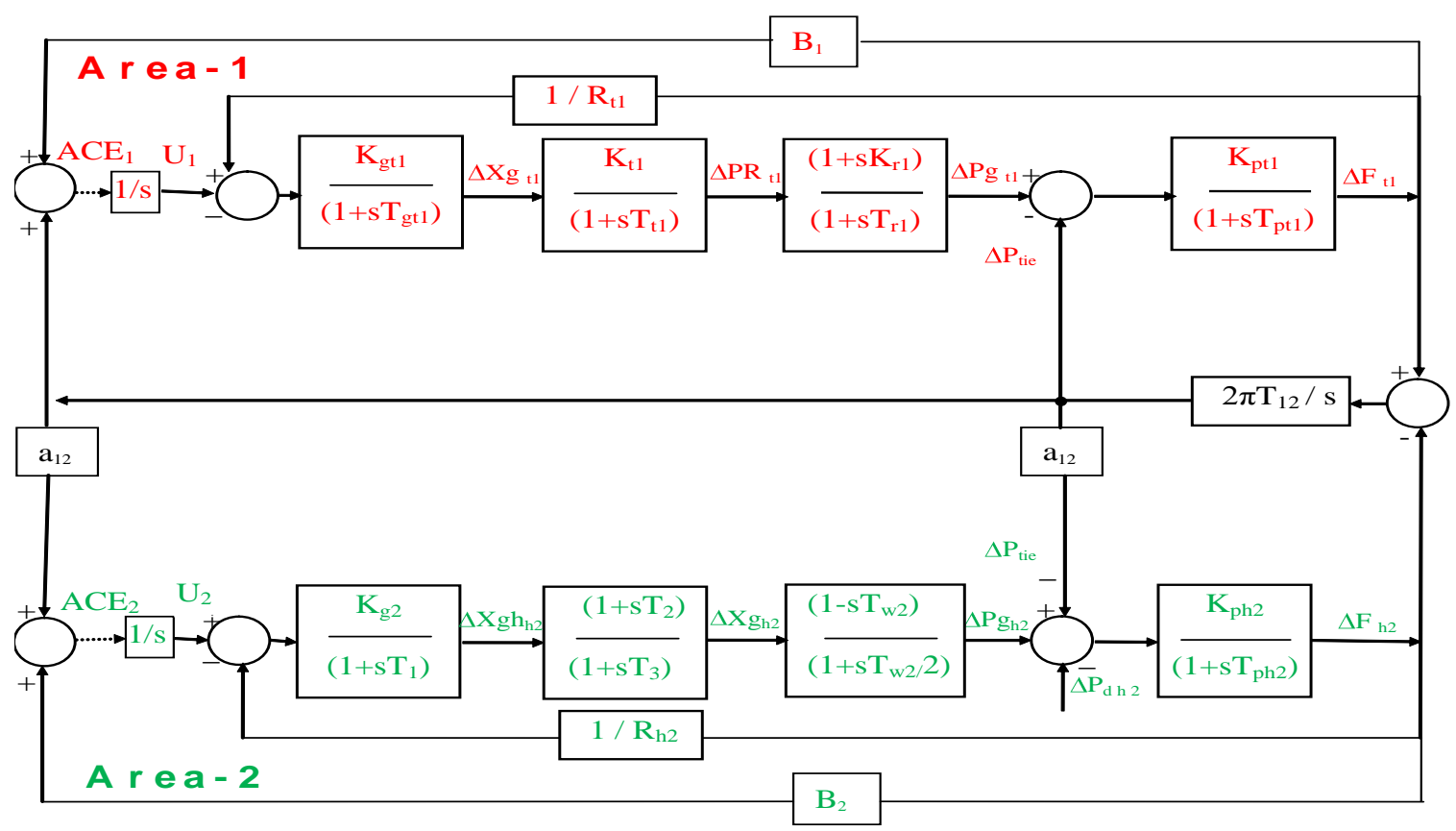

Fig.4 Transfer Function Model of Interconnected Hydro-Thermal Power System 